\title{
Hydrogen Combustion in I.C Engines
}

\author{
Ast. Prof B.W.Sandeep Kumar, Aravindakshan.R, Gowri Shankar.K, \\ Kannan.C.R, Prabhakaran.S \\ Srm University \\ Srm University
}

\begin{abstract}
The use of hydrogen as a fuel for the internal combustion engine represents an alternative to solve both of the greatest problems related to the use of classic fuels: the limitation of the fossil fuels consumption and the environment pollution. We first describe hydrogen-engine fundamentals by examining the engine-specific properties of hydrogen and topics that are discussed include abnormal combustion (backfire, pre-ignition and knock), emissions of NOx and load control strategies (power output versus NOx tradeoff). Finally, we consider comprehensive overview of the design features of a dedicated hydrogen S.I., Direct-Injection hydrogen fueled internal combustion engine.
\end{abstract}

\section{Introduction:}

At the moment the estimation of the number of motor vehicles is about 800 million. To replace them in a relatively short time by fuel cells is impossible. There are several reasons for converting the gasoline, diesel or natural gas engines to hydrogen fuelled internal combustion engines. ICEs are proven technology, are simple and well-known and the adaptations can be made with a low cost. During the transition period bi-fuel solutions are possible (to run the engine either on gasoline or pure hydrogen). For larger engines (buses, trucks) mixtures of natural gas with hydrogen (about 20\%) are easy to exploit. During this transition period, experience can be gained with the production, storage and infrastructure of hydrogen. Currently the hydrogen production is the cheapest through the steam reforming of methane. But $\mathrm{CO} 2$ emissions can not be avoided. Renewable energy, e.g. solar power, hydroelectric, tidal, ... can give - CO2-freell electricity to electrolyze water to hydrogen. The downside is that these electricity costs are mostly expensive. Interesting is also the application of peak shaving of wind turbine power. Other possibilities are solar thermal, biomass, bacterial...

The unique combustion characteristics of hydrogen that allow clean and efficient operation at low engine loads present difficulties at high engine loads. Here, the low ignition energies of hydrogen-air mixtures cause frequent unscheduled combustion events, and high combustion temperatures of mixtures closer to the stoichiometric composition lead to increased NOx production. Both effects, in practical application, limit the power densities of H2ICEs. The recent research thrust and progress on this front is the development of advanced hydrogen engines with improved power densities and reduced NOx emissions at high engine loads.

\section{H2ICE Fundamentals:}

The ability for H2ICEs to burn cleanly and operate efficiently is owed to the unique combustion characteristics of hydrogen that allow ultra-lean combustion with dramatically reduced NOx production and efficient low-engine load operation. In contrast, the same combustion characteristics impose technical challenges at high engine-loads due to an increased propensity to preignite the hydrogen-air mixture and increased NOx production. In this section we review the benefit and technical challenge of H2ICE operation at low and high engine-load, through an examination of hydrogen properties relevant to engine operation and control. Hydrogen properties of interest are listed in Table 1 [1]. The same properties for typical gasoline and compressed natural gas (CNG) are also provided. For direct comparison, H2ICE engine properties will be compared to those of a PFI gasoline engine.

FUEL PROPERTIES AT 298K, 1 ATM ;

\begin{tabular}{|l||l|l|l|l||}
\hline $\begin{array}{l}\text { Property Hydrogen } \\
\text { Gasoline }\end{array}$ & & & \\
\hline Density(kg/m3) & 0.0824 & 0.72 & $4.3-15$ & 730 \\
Flammability limits(volume \% in & $4-75$ & & $0.4-7.6$ & \\
Autoignition temp in air(K) & $0.1-7.1$ & $0.7-4$ & \\
\hline
\end{tabular}




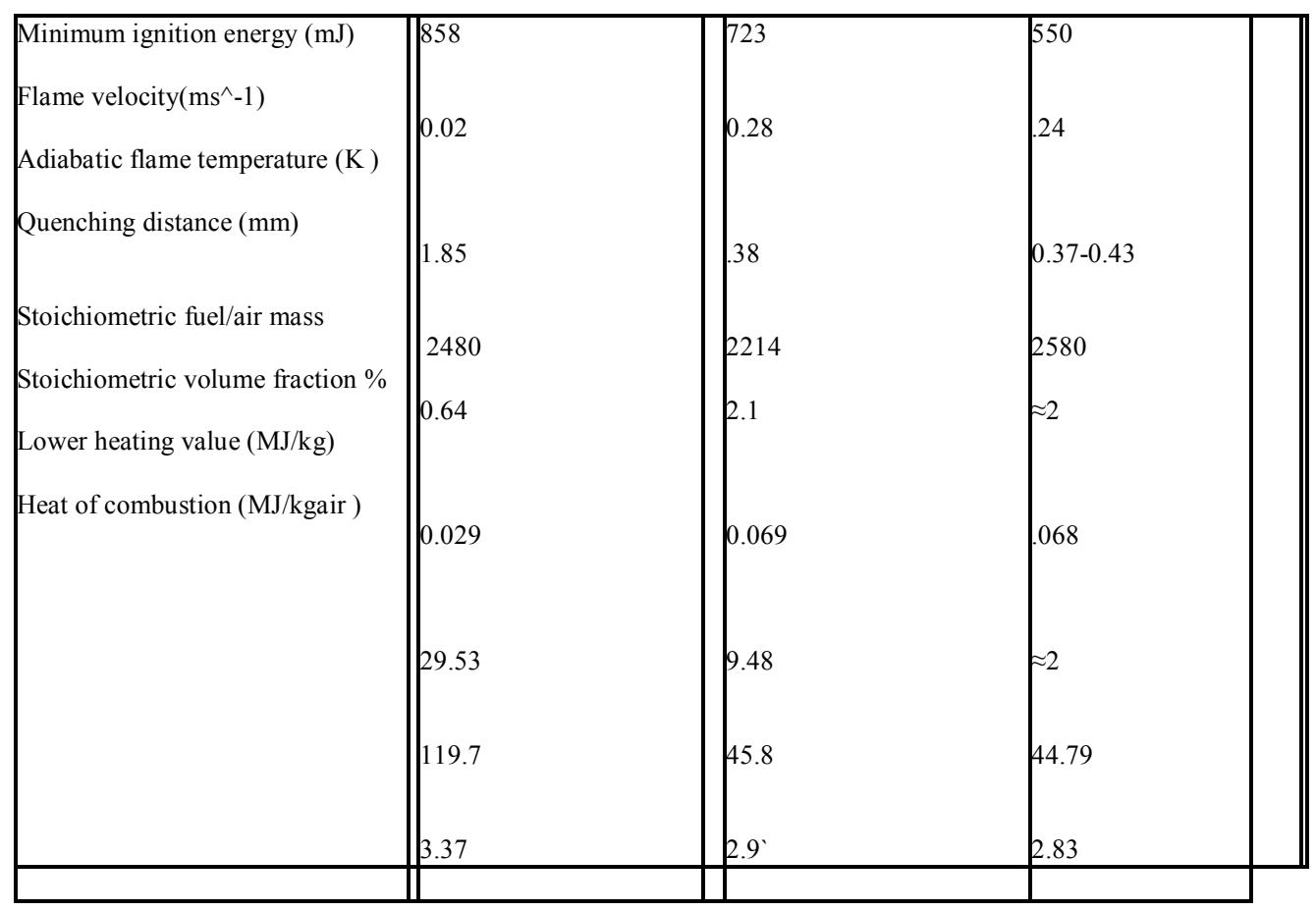

\subsection{Preignition and knock:}

The high autoignition temperature of hydrogen $(858 \mathrm{~K})$ means that hydrogen is most suitable as a fuel for spark ignition (SI) engines. Despite the high autoignition temperature, the ignition energies of hydrogen-air mixtures are approximately an order-of-magnitude lower than that of hydrocarbon-air mixtures. This is observed in Fig. 1 where the minimum ignition energies for hydrogen-air, propane-air and heptane-air mixtures at atmospheric pressure are plotted as a function of equivalence ratio, $\Phi$, where $\Phi$ is defined as the ratio of the actual fuel/air mass ratio to the stoichiometric fuel/air mass ratio. The low ignition energies of hydrogen-air mixtures mean that H2ICEs are predisposed towards the limiting effects of preignition. Here, preignition is defined as combustion prior to spark discharge, and in general, results from surface ignition at engine hot spots, such as spark electrodes, valves or engine deposits. The limiting effect of preignition is that a preignition event will advance the start of combustion and produce an increased chemical heat release rate. In turn, the increased heat-release rate results in a rapid pressure rise, higher peak cylinder pressure, acoustic oscillations and higher heat rejection that leads to higher in-cylinder surface temperatures. The latter effect can advance the start of combustion further, which in turn can lead to a runaway effect, and if left unchecked will lead to engine failure.It is therefore a necessity for practical application that preignition is avoided.

Fig. 1. Minimum ignition energies of $(\cdot)$ hydrogen-air, ( $\bullet$ ) methane-air and () heptane-air mixtures in relation to $\varphi$ at atmospheric pressure

Knock, or spark knock, is defined as autoignition of the hydrogen-air end-gas ahead of the flame front that has originated from the spark. The high autoignition temperature, finite ignition delay and the high flame velocity of hydrogen (i.e., the latter two effects translate to less residence time for the end-gas to ignite) means that knock, as defined, is less likely for hydrogen relative to gasoline, and hence the higher research octane number (RON) for hydrogen (RON>120) in comparison to gasoline (RON9199) [2].

The global effect of both knock and preignition (i.e., an audible pinging or - knockingl) is nearly indistinguishable. However, a distinction is necessary since the controlling phenomena are very different: preignition can be avoided through proper engine design, but knock is an inherent limit on the maximum compression ratio that can be used with a fuel. The differentiating factor between knock and preignition is that knock can be controlled by retarding the spark timing, while, in general, preignition cannot be controlled by adjusting spark timing.

Observed in Fig. 1 is that as the stoichiometric condition $(\Phi 1)$ is approached from the lean side $(\Phi<1)$, the minimum ignition energy for hydrogen is a strongly decreasing function of the equivalence ratio with the minimum at $\Phi 1$. This trend is responsible for the experimentally observed fact that it is extremely difficult to operate an H2ICE at or near the stoichiometric condition in the absence of frequent pre-ignition events. Therefore, for practical application, the maximum $\Phi$ and, consequently, peak power 6 output can be limited by the pre-ignition-limit. Stockhausen et al. Report a pre-ignition-limit of $\Phi 0.6$ for a 4-cylinder engine at an engine 
speed of $5000 \mathrm{rpm}$. Consequently, engine peak power output was reduced by $50 \%$ compared to engine operation with gasoline.

\subsubsection{Preignition control:}

The diminished peak power output, set by the preignition-limit, will decrease the performance of a H2ICE-powered vehicle in comparison to its gasoline equivalent. Therefore, determining the mechanism of preignition, practical operational limits, and control strategies has been a primary focus of many research studies.Unfortunately, despite much effort, there exist no guaranteed preventive steps. However, identification of preignition sources, such as incylinder hot-spots, oil contaminants, combustion in crevice volumes and residual energy [4],in the ignition system, has provided the necessary minimizing steps. These include use of cold-rated spark plugs, low coolant temperature and optimized fuel-injection timing.

Advanced control strategies include intake charge cooling, variable valve timing for effective scavenging of exhaust residuals, advanced ignition systems, and hydrogen direct injection (DI). These advanced strategies can be quite effective using a single-cylinder 0.5-1 PFI-H2ICE at CR of 12:1 used variable cam phasing and computational fluid dynamics (CFD) simulations to optimize coolant flow, injection location, and injection timing to operate at $\Phi 1$ over a speed range 2000-4000 rpm. Homan et al. [5] used DI late in the compression stroke and near-simultaneous spark to eliminate preignition events. However, to prevent misfires, the hydrogen jet had to be directed towards the spark. Kondo et al. [4] used an ignition system specifically designed to prevent residual energy and a water cooled spark plug. In the absence of any advanced control, maximum $\Phi 0.35$; by the elimination of residual energy in the ignition system, maximum $\Phi 0.6$; with the addition of the water-cooled spark plug, maximum $\Phi 0.8$. With liquid-hydrogen fueling, report that preignition can be eliminated without too much effort simply due to the cooling effect of the cold hydrogen.

\subsection{Flammability range, flame velocity and adiabatic flame temperature:}

The flammability range, in fuel volume fraction in air at $298 \mathrm{~K}$ and $1 \mathrm{~atm}$, for hydrogen is $4-75 \%$ and for gasoline is $1-7.6 \%$. For ICEs, in terms of equivalence ratio. Then, the flammability range of hydrogen is $0.1<\Phi<7.1$, and gasoline is approximately $0.7<\Phi_{-}<4$. In recasting the flammability range in terms of equivalence ratio, it is evident that the H2ICE is amenable to stable operation under highly dilute conditions, which allows more control over engine operation for both emissions reduction and fuel metering. In practical application, the diluent can either be excess air (lean operation) or recycled exhaust gas. An important distinction between the two is that the latter allows stoichiometric operation at low to medium load and the use of a three-way catalyst (TWC) for NOx reduction. However, problems due to water condensation using recycled exhaust gas dilution in ICEs is exacerbated in H2ICEs due to the large fraction of $\mathrm{H} 2 \mathrm{O}$ in the exhaust stream.

Flame velocity and adiabatic flame temperature are important properties for engine operation and control, in particular thermal efficiency, combustion stability and emissions. Laminar flame velocity and flame temperature, plotted as a function of equivalence ratio, are shown in Figs. 2 and 3, respectively.

Fig. 2. Laminar flame velocity for (-) hydrogen, oxygen and nitrogen mixtures and (, - -) gasoline and air mixtures at room temperature and atmospheric pressure. The dashed line is a least squares fit polynomial.[OO2]/[O2+N2] is the oxygen mole fraction in the ambient.

Fig. 3. Adiabatic flame temperature for hydrogen-air mixtures

\subsection{Nitrogen oxides (NOx):}

There is a tradeoff between H2ICE power output and NOx emissions. Considering that the primary benefit of an H2ICE is near-zero emissions, a practical limit of engine operation is $\Phi<0.5$ (assuming no EGR). This restriction will translate to a large loss in the effective power density of an H2ICE. The potential to expand the power band while maintaining near-zero NOx emissions is possible by charge dilution with EGR and use of a TWC or by improving lean power density with pressure boosting.

Exhaust gas recirculation is an effective means for NOx reduction and an especially interesting option at stoichiometric operation as the high NOx reduction efficiency of a standard three way catalyst can then be exploited. Furthermore, one could vary the engine power output by changing the amount of recycled exhaust gas, instead of throttling, thus avoiding engine efficiency penalties.

A comparison was made between lean burn operation and stoichiometric operation with EGR, in terms of efficiency, power output, NOx emissions and backfire resistance. Figs. 4 and 5 compare the lean burn with the EGR load control strategy. Fig. 4 shows the NOx emissions before and after the TWC as a function of the air to fuel equivalence ratio (here, the power output is controlled by the equivalence ratio). Fig. 5 plots the NOx emissions before and after the TWC as a function of the percentage of EGR (here, the power output is controlled by the EGR percentage, while maintaining a stoichiometric mixture). Only at stoichiometric or at rich conditions, there is enough hydrogen present in the exhaust to act as an active agent to reduce the NOx. Figure 5 
learns that the conversion efficiency of the TWC decreases with the EGR percentage, which is probably due to the exhaust temperature dropping below the lightoff temperature of the TWC.

Figure 4: NOX emissions before and after TWC, TWC, stoichiometric

As a funtion of the equivalence ratio
Figure 5: NOX emissions before and after

operation variable EGR percentage

\subsection{Load Control Strategies:}

Hydrogen is a very versatile fuel when it comes to load control. The high flame speeds of hydrogen mixtures and its wide flammability limits permit very lean operation and substantial dilution. The engine efficiency and the emission of NOx are the two main parameters used to decide the load control strategy. Constant equivalence ratio throttled operation has been used but mainly for demonstration purposes, as it is fairly easy to run a lean burn throttled hydrogen engine (accepting the severe power output penalty). Where possible, wide open throttle (WOT) operation is used to take advantage of the associated increase in engine efficiency, regulating load with mixture richness (qualitative control) instead of volumetric efficiency (quantitative control) and thus avoiding pumping losses. Limitations to WOT operation are due to misfires, unburned hydrogen and decreased stability at very low load (e.g. idling) and NOx emissions at medium to full load. Thus, throttling is used at very low loads to increase combustion stability and decrease unburned hydrogen emissions . Moreover, this increases the efficiency at these conditions: the efficiency gain through decrease of unburned hydrogen emissions offsets the efficiency loss by throttling. The engine efficiency using throttled or WOT operation is compared, the lean limit at which throttling is introduced is engine dependent and ranges from $\Phi=0.33$ to $\Phi=0.25$. For higher loads, flame temperatures quickly exceed the NOx generation limit. This results in a NOx limit to WOT operation. One could restrict the mixture richness and use sufficiently lean mixtures to stay below a 10 or 100 ppm NOx limit, but this implies a large decrease in maximum power output. Alternatively, the engine can be throttled above this limit, using stoichiometric mixtures and thus enabling the use of a conventional three way catalyst for NOx reduction, with an associated decrease in engine efficiency. Yet another strategy is the use of EGR to control load: using stoichiometric mixtures but instead of throttling, recycling exhaust gas in a proportion dependent on the power demand. This gives a better efficiency compared to throttling. Water injection can also be used to decrease NOx emissions from the richer mixtures, and is more effective than EGR but is impractical.

\section{Overview Of Design Features Of Dedicated Hydrogen Si Engines:}

An overview was given of the development by car manufacturers, research centers and also of the research at the laboratory of Transport Technology, Ghent University. This can be synthesized in the following overview of the design features in which a dedicated hydrogen engine differs from traditionally fueled engines:

Spark plugs: use cold rated spark plugs to avoid spark plug electrode temperatures exceeding the auto-ignition limit and causing backfire[6]. Cold rated spark plugs can be used since there are hardly any spark plug deposits to burn off. Do not use spark plugs with platinum electrodes as this can be a catalyst to hydrogen oxidation (platinum has been used in the exhaust to oxidize unburned hydrogen ).

\section{Ignition system:}

avoid uncontrolled ignition due to residual ignition energy by properly grounding the ignition system or changing the ignition cable's electrical resistance [4]; avoid induction ignition in an adjacent ignition cable, for instance by using a coil-on-plug system; provide a high voltage output ignition system as the ignition of hydrogen mixtures asks for an increased secondary ignition voltage [4] (probably because of the lower ion concentration of a hydrogen flame, see above), coil on- plug systems also satisfy this condition. Alternatively, the spark plug gap can be decreased to lower the ignition voltage, this is no problem for hydrogen engines as there will be almost no deposit formation. Spark plug gaps as small as $0.25 \mathrm{~mm}$ have been used (although the gap was subsequently increased to $0.5 \mathrm{~mm}$ because of cold start difficulties due to water condensation at the spark plug tip).

Injection system: provide a timed injection, either using port injection and programming the injection timing such that an initial air cooling period is created in the initial phase of the intake stroke and the end of injection is such that all hydrogen is inducted, leaving no hydrogen in the manifold when the intake valve closes; or using direct injection during the compression stroke. High flow rate injectors are needed in both cases, multiple injectors per cylinder can alleviate this requirement. The timing described here might not be necessary as work has been reported where no relation between injection timing and backfire or pre-ignition limited equivalence 
ratio was found. Timed injection also decreases the amount of unburned fuel in the intake manifold at any given time, limiting the severity of a backfire should it occur.

Hot spots: avoid hot spots in the combustion chamber that could initiate pre-ignition or backfire, use cooled exhaust valves; use multi-valve engine heads to further lower the exhaust valve temperature ; ensure proper oil control; provide additional engine coolant passages around valves and other areas with high thermal loads (if possible); delay fuel introduction to create a period of air cooling (using timed manifold or direct injection); ensure adequate scavenging (e.g. using variable valve timing) to decrease residual gas temperatures.

Piston rings and crevi ce volumes: decrease the piston top land clearance to prevent hydrogen flames from propagating into the top land, use a clearance of $0.152 \mathrm{~mm}$ to quench the hydrogen flame. Change the crevice volumes and/or piston rings with the aim of reducing the reflow of unburned mixture from the second land to the top land (preventing_fuelling 'of a top land flame during exhaust and intake)

In-cylinder turbulence: because of the high flame speeds of hydrogen, low turbulence combustion chambers can be used which are beneficial for the engine efficiency. They might even be necessary to avoid abnormal combustion at stoichiometric operation .

Compression ratio: the choice of the optimal compression ratio is similar to that for any fuel, it should be chosen as high as possible to increase engine efficiency, with the limit given by increased heat losses or appearance of abnormal combustion The choice may depend on the application, as the optimum compression ratio for highest engine efficiency might be different from the optimum for highest power output. In general, the compression ratio of a hydrogen engine can be chosen higher than that for a gasoline engine.

\subsection{Direct-injection hydrogen-fueled internal combustion engine (DI-H2ICE):}

The direct injection H2ICE has long been viewed as one of the most attractive advanced H2ICE options. The view is based on the high volumetric efficiency, (since hydrogen is injected after intake valve closing) and the potential to avoid preignition. The latter effect is controlled by timing injection to both minimize the residence time that a combustible mixture is exposed to in-cylinder hot spots (i.e., late injection) and allow for improved mixing of the intake air with the residual gases. The improved volumetric efficiency (equal to PFI gasoline or higher) and the higher heat of combustion of hydrogen compared to gasoline provides the potential for DI-H2ICE power density to be approximately $115 \%$ that of the identical engine operated on gasoline. In particular a $15 \%$ increase in IMEP for engine operation with DI hydrogen compared to engine operation with PFI gasoline.

For early injection (i.e., coincident with IVC) maximum available mixing times range from approximately 20-4 ms across the speed range 1000-5000 rpm, respectively. In practice, to avoid preignition, start of injection (SOI) is retarded with respect to IVC, and mixing times are further reduced. To evaluate the plausibility of complete mixing in a DI-H2ICE, using experimental correlations for air-entrainment rates in free turbulent jets and order of magnitude expressions for turbulent mixing times, estimated that a free-hydrogen jet with sonic velocity at the orifice issuing into air will entrain a stoichiometric amount of air in approximately 1 ms. However, contrary to this optimistic estimate, the overwhelming experimental evidence demonstrates that complete mixing in an engine takes approximately $10 \mathrm{~ms}$. conjectured that the order-of-magnitude difference in mixing times between the estimate for a free jet and that measured in an engine is a result of fluid flow interaction between in-cylinder flow and the hydrogen jet. A strong interaction will cause the free-jet analysis to break down. As evidence, Homan et al. [4] demonstrated, in an engine with side injection and side spark (separated by 180), that the frequency of misfire for late injection decreased significantly when the injected hydrogen was in coflow with the swirl instead of in crossflow.In order to take full advantage of the potential of the DI-H2ICE, a high-pressure, high flow-rate hydrogen injector is required for operation at high engine speeds and to overcome the in-cylinder pressure for injection late in the compression stroke. Here we define high pressure as greater than 80 bar to ensure sonic injection velocities and high enough mass flow rates for SOI throughout the compression stroke. The need for rapid mixing necessitates the use of critical flow injectors and the short time duration with late injection requires high mass flow rates. To prevent valve leakage at the valve seat a seal made of an elastomer material has been used with success. Typical flow rates required are $1-10 \mathrm{~g} \mathrm{~s} 1$.

\section{Conclusions:}

This paper presents a comprehensive overview of hydrogen engine properties and design features. An important conclusion from the work described above is that the hydrogen ICE is rapidly gaining on traditionally fueled engines and is a serious competitor for fuel cells. Hydrogen ICEs have a high efficiency, are very clean and are considerably cheaper than fuel cells. 
For further improvements in power output and prevention of abnormal combustion, it would be interesting to perform a systematic study of the =knocking' behavior and crevice combustion to obtain a better understanding of the mechanisms leading to abnormal combustion phenomena such as backfire. More specifically, data is needed of the knocking behavior and the quenching distance as a function of the equivalence ratio.

Advanced H2ICE options requires: continued advancements in fundamental H2ICE research, reduction of NOx formation and emissions, research and development of advanced engine components, and highly advanced control and optimization strategies.

\section{References:}

[1]. Drell IL, Belles FE. Survey of hydrogen combustion properties.

[2]. Technical Report 1383. National Advisory Committee on Aeronautics, 1958.

[3]. Stockhausen WF, Natkin RJ, Kabat DM, Reams L, Tang X, Hashemi S, et al. Ford P2000 hydrogen engine design and vehicle development program. SAE paper 2002; 2002-01-0240.

[4]. Kondo T, Lio

[5]. Homan HS, DE Boer PCT, McLean WJ. 